Tropical Journal of Pharmaceutical Research June 2016; 15 (6): 1299-1305

ISSN: $1596-5996$ (print); 1596-9827 (electronic) (C) Pharmacotherapy Group, Faculty of Pharmacy, University of Benin, Benin City, 300001 Nigeria.

All rights reserved.

\title{
Identification of metabolites of kurarinone from Sophora flavescens Ait in rat urine by ultra-performance liquid chromatography with linear ion trap orbitrap mass spectrometry
}

\author{
Yi Liư ${ }^{1}$, Zhi Xian Mo ${ }^{1}$, Chun Guo Wang ${ }^{2}$, Rong Huang ${ }^{3}$, Feng Wang ${ }^{3}$ and Lei \\ Chen $^{3 *}$ \\ ${ }^{1}$ School of Chinese Medicine, Southern Medical University, Guangzhou 510006, ${ }^{2}$ Center of Scientific Experiment, Beijing \\ University of Chinese Medicine, Beijing $100029,{ }^{3}$ College of Traditional Chinese Materia Medica, Guangdong Pharmaceutical \\ University, and Key Unit of Chinese Medicine Digitalization Quality Evaluation of SATCM, Guangzhou 510006, China
}

*For correspondence: Email: chenlei0080@163.com; Tel: +86-20-39352177

Received: 8 January 2016

Revised accepted: 10 May 2016

\begin{abstract}
Purpose: To study the in vivo metabolism of kurarinone, a lavandulyl flavanone which is a major constituent of Kushen and a marker compound with many biological activities, using ultra-performance liquid chromatography coupled with linear ion trap Orbitrap mass spectrometry (UPLC-LTQ-OrbitrapMS).

Methods: Six male Sprague-Dawley rats were randomly divided into two groups. First, kurarinone was suspended in $0.5 \%$ carboxymethylcellulose sodium (CMC-Na) aqueous solution, and was given to rats ( $n=3,2 \mathrm{~mL}$ for each rat) orally at $50 \mathrm{mg} / \mathrm{kg}$. A $2 \mathrm{~mL}$ aliquot of $0.5 \% \mathrm{CMC}-\mathrm{Na}$ aqueous solution was administered to the rats in the control group. Next, urine samples were collected over 0-24 $h$ after the oral administrations and all urine samples were pretreated by a solid phase extraction (SPE) method. Finally, all samples were analyzed by a UPLC-LTQ-Orbitrap mass spectrometry coupled with an electrospray ionization source (ESI) that was operated in the negative ionization mode.

Results: A total of 11 metabolites, including the parent drug and 10 phase II metabolites in rat urine, were first detected and interpreted based on accurate mass measurement, fragment ions, and chromatographic retention times. The results were based on the assumption that kurarinone glucuronidation was the dominant metabolite that was excreted in rat urine.

Conclusion: The results from this work indicate that kurarinone in vivo is typically transformed to nontoxic glucuronidation metabolites, and these findings may help to characterize the metabolic profile of kurarinone.
\end{abstract}

Keywords: Kurarinone, Metabolites, Sophora flavescens Ait., Glucuronidation metabolites

Tropical Journal of Pharmaceutical Research is indexed by Science Citation Index (SciSearch), Scopus, International Pharmaceutical Abstract, Chemical Abstracts, Embase, Index Copernicus, EBSCO, African Index Medicus, JournalSeek, Journal Citation Reports/Science Edition, Directory of Open Access Journals (DOAJ), African Journal Online, Bioline International, Open-J-Gate and Pharmacy Abstracts

\section{INTRODUCTION}

Sophora flavescens Ait (Leguminosae) is a perennial shrub that is widely distributed in Asia, Oceania, and the Pacific Islands. For thousands of years, the dried roots of Sophora flavescens, also named 'Kushen', have been traditionally used in East Asian countries as an herbal medicine for the treatment of hepatitis, gastrointestinal hemorrhage and skin diseases.

Phytochemical studies of Sophora flavescens described the isolation of quinolizidine alkaloids, 
flavonoids and triterpenoids. The alkaloids in Sophora flavescens mainly consist of oxymatrine and matrine, which have been used clinically in China for the treatment of chronic hepatitis. Furthermore, several pharmacological products, such as injection oxymatrine and capsule matrine, are extensively used for the treatment of hepatitis and cancers in China. The flavonoids, which have been termed Kushen flavonoids, usually carry one or multiple prenyl units. Several studies have demonstrated that prenyl chains are crucial for the biological activity of flavonoids [1]. Therefore, the pharmacology of Kushen prenylated flavonoids has become an area of increased research because of their anti-diabetic, anti-arthritic, anti-inflammation, and anti-bacterial activities.

Kurarinone, a lavandulyl flavanone, is a major constituent of Kushen flavonoids, and it has a concentration range of $4.79-16.07 \mathrm{mg} / \mathrm{g}$ [2]. It is considered to be a marker compound with many biological activities, such as anti-immune [3], anti-bacterial [4], anti-atherosclerosis [5], anticancer [6], anti-carcinogenic [7] activities. Furthermore, some studies have suggested that kurarinone in combination with TRAIL has potential application for the treatment of tumors and gastric cancer [8], and it can also potentially increase the antitumor activity of Taxol [9].

Although kurarinone has various pharmacological effects, studies on the in vivo metabolism of kurarinone have rarely been reported. Yu [10] demonstrated that kurarinone induced remarkable cytotoxicity in primary rat hepatocytes and $\mathrm{HL}-7702$ cells, with $\mathrm{IC}_{50}$ values of 29.9 and $48.2 \mu \mathrm{M}$, respectively. The metabolism of kurarinone by human liver microsomes in vitro indicated that kuarinone could be extensively glucuronidated to decrease its cytotoxicity [11].

Metabolism, which plays an important role in pharmacokinetics, can influence the biological activity and toxicity of drugs [12]. Further studies of kurarinone metabolism and the role of metabolism in biological activity or toxicity should be performed to provide a comprehensive overview of the clinical use of kurarinone.

\section{EXPERIMENTAL}

\section{Isolation and identification of kurarinone}

Kurarinone (Figure 1) was isolated in our laboratory from the ethanol extract of Sophora flavescens Ait.. A $5 \mathrm{~kg}$ quantity of dry kushen roots was extracted three times with ethanol. The ethanol extract was evaporated to dryness, and the dry residue was suspended in water and further partitioned in succession with ethyl acetate and butanol. Part of the ethyl acetate extractives was chromatographed over $1000 \mathrm{~g}$ of a silica gel column (200 - 300 mesh) using a $\mathrm{CH}_{2} \mathrm{Cl}_{2}-\mathrm{MeOH}$ step gradient elution, yielding ten fractions. The fractions (NO. 6) were chromatographed on MCl GEL CHP20P using a $\mathrm{MeOH}-\mathrm{H}_{2} \mathrm{O}$ step gradient elution, and they were purified further by preparative HPLC (C18) to yield kurarinone $(3.01 \mathrm{~g})$. Its structure was elucidated by the comprehensive interpretation of the ESI - MS and ${ }^{1} \mathrm{H},{ }^{13} \mathrm{C}$ NMR data. The kurarinone purity was higher than $99 \%$ according to HPLC - UV analysis.

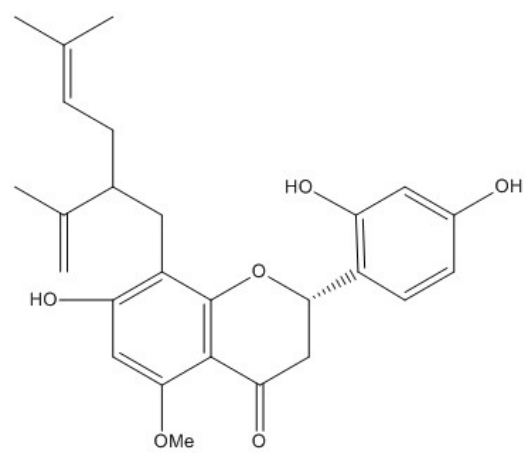

Figure 1: Chemical structures of kurarinone

\section{Chemicals and materials}

HPLC grade acetonitrile, methanol, and formic acid were purchased from Fisher (NJ, USA). Ultra-pure water was prepared using a Milli-Q water purification system (Millipore, Billerica, MA, USA). Waters Oasis HLB solid-phase extraction (SPE) cartridges ( $3 \mathrm{cc} / 60 \mathrm{mg}, 30 \mu \mathrm{m}$ ) were used. All other chemicals were of analytical grade and they were commercially available.

\section{Animal experiments and drug administration}

Six male Sprague-Dawley rats $(200 \pm 10 \mathrm{~g})$ were provided by Southern Medical University Experimental Animals Center (Guangzhou, China). Sprague-Dawley rats were housed in controlled environmental conditions (temperature: $24 \pm 2{ }^{\circ} \mathrm{C}$; relative humidity: $70 \pm 5$ $\%)$. They were housed individually for a week for acclimatization, and they were fed ad libitum with standard laboratory chow (Southern Medical University Experimental Animals Center, Guangzhou China) and water. All the rats were fasted for more than $12 \mathrm{~h}$ with free access to water before the experiment. The rats were randomly divided into two groups: Group A ( $\mathrm{n}=$ $3)$, drug group for urine; Group $B(n=3)$, control group for blank urine. All experimental protocols involving animals and their care were approved 
(ref. no. SYXK (YUE) 2011 - 0015) by the institutional Animal Care and Use Committee at Southern Medical University (Guangzhou, China), and the experimental protocols followed the "Guide for the Care and Use of Laboratory Animals" [13].

Kurarinone $(10 \mathrm{mg} / \mathrm{mL})$ was suspended in $0.5 \%$ carboxymethylcellulose sodium (CMC-Na) aqueous solution. The rats in the drug group were orally exposed to kurarinone at a dose of $50 \mathrm{mg} / \mathrm{kg}$ body weight. Next, a $2 \mathrm{~mL}$ aliquot of $0.5 \%$ CMC-Na aqueous solution was administered to the rats in the control group. Urine samples were collected over a period of 0 $24 \mathrm{~h}$ after the oral administrations, and all the biological samples from the same group were pooled into one sample.

\section{Sample preparation}

All the rat urine samples were pretreated by a solid-phase extraction (SPE) method. A preactivated Oasis HLB solid phase extraction C18 column was loaded with a $1 \mathrm{~mL}$ urine sample, and then the cartridge was eluted by $5 \mathrm{~mL}$ water and $5 \mathrm{~mL}$ methanol successively. The methanol eluate was collected and evaporated to dryness by $\mathrm{N}_{2}$. The residues were reconstituted in $100 \mu \mathrm{L}$ methanol and then centrifuged at 14,000 rpm for $15 \mathrm{~min}$ at $4{ }^{\circ} \mathrm{C}$. The final $2 \mu \mathrm{L}$ solution was injected into an UPLC-ESI-LTQ-Orbitrap MS for analysis.

\section{Instruments and conditions}

A Finnigan LTQ/Orbitrap MS (Thermo Electron, Bremen, Germany) coupled to an ESI source (Thermo Electron, Bremen, Germany) was used for all samples analysis. A Waters ACQUITY $\mathrm{BEH}$ C18 column $(2.1 \times 100 \mathrm{~mm}$ i.d., $1.7 \mu \mathrm{m})$ was used for separation of the metabolites at room temperature and a flow rate of $200 \mu \mathrm{L} / \mathrm{min}$. A linear gradient elution using $0.5 \%$ formic acid aqueous solution (A) and acetonitrile (B) was applied with the following program: 0 - $3 \mathrm{~min}, 3$ $5 \%$ B; 3 - 4 min, 5-30\% B; 4 - 20 min, 30 - $36 \%$ B; 20 -21 min, 36 - $42 \%$ B; 21 - 22 min, 42 - 60 $\%$ B; 22 - $25 \min , 60-61 \%$ B; 25 - $26 \min , 61$ $80 \% \mathrm{~B} ; 26-30 \mathrm{~min}, 80 \% \mathrm{~B} ; 30-31 \mathrm{~min}, 80-3$ $\% \mathrm{~B}$, and $31-35 \mathrm{~min}, 3 \% \mathrm{~B}$.

An aliquot of $2 \mu \mathrm{L}$ was injected into the UPLCLTQ Orbitrap MS. The optimized operating parameters in the negative ionization mode were as follows: capillary voltage of $35 \mathrm{~V}$, electrospray voltage of $3.0 \mathrm{kV}$, capillary temperature of 350 ${ }^{\circ} \mathrm{C}$, sheath gas flow rate of 30 (arbitrary units), auxiliary gas flow rate of 10 (arbitrary units), and tube lens voltage of $110 \mathrm{~V}$.
Metabolites were detected by full scan massanalysis from m/z 100 to 1000 at a resolving power of 30,000 with data-dependent $\mathrm{MS}^{2}$ analysis triggered by the three most-abundant ions from the precursor list of predicted metabolites followed by $\mathrm{MS}^{3}$ analysis of the most abundant product ions. Collision-induced dissociation (CID) was performed with an isolation width of $2 \mathrm{Da}$. The collision energy was set to $35 \%$.

\section{Peak selection and data processing}

Thermo Xcalibur 2.1 workstation (Thermo Scientific, Bremen, Germany) was used for data acquisition and processing. To obtain the maximum number of fragments, the peaks detected with intensity $>10000$ were selected for identification. The chemical formulas for all parent ions of the selected peaks were calculated from the accurate mass using a formula predictor by setting the parameters as follows: C [0 - 40], H [0 - 50], O [0 - 20], S [0 - 4], $\mathrm{N}[0-4], \mathrm{Cl}[0-4]$, and ring double bond (RDB) equivalent value [0 - 15]. Other elements such as $\mathrm{P}$ and $\mathrm{Br}$ were not considered, as they were rarely present in the complex matrix. Blank biological samples were used as controls to compare with the analyte samples, and they were processed under the same conditions.

\section{RESULTS}

\section{Fragmentation pathway of kurarinone}

First, the $\mathrm{MS}^{\mathrm{n}}$ fragmentation pattern of kurarinone was investigated to facilitate the structural identification of the metabolites. The parent ion showed a deprotonated ion $[\mathrm{M}-\mathrm{H}]^{-}$at $\mathrm{m} / \mathrm{z} 437.1950$ (2.0 ppm, $\mathrm{C}_{26} \mathrm{H}_{29} \mathrm{O}_{6}$ ) in negative ionization mode. The fragmentation of this parent ion provided a characteristic fragment ion at $\mathrm{m} / \mathrm{z}$ $275\left(\mathrm{C}_{17} \mathrm{H}_{23} \mathrm{O}_{3}\right)$ by the loss of the $\mathrm{C}_{9} \mathrm{H}_{6} \mathrm{O}_{3}$ moiety. The proposed fragmentation pattern of kurarinone is illustrated in Figure 2.

\section{Metabolites of kurarinone in rats}

After comparing the total ion chromatogram of the drug samples with the corresponding control samples, a total of 10 metabolites and the parent drug were detected and identified. The total ion chromatogram of the drug samples is shown in Figure 3. The chromatographic and mass spectrometric data of the parent drug and its metabolites are shown in Table 1. 


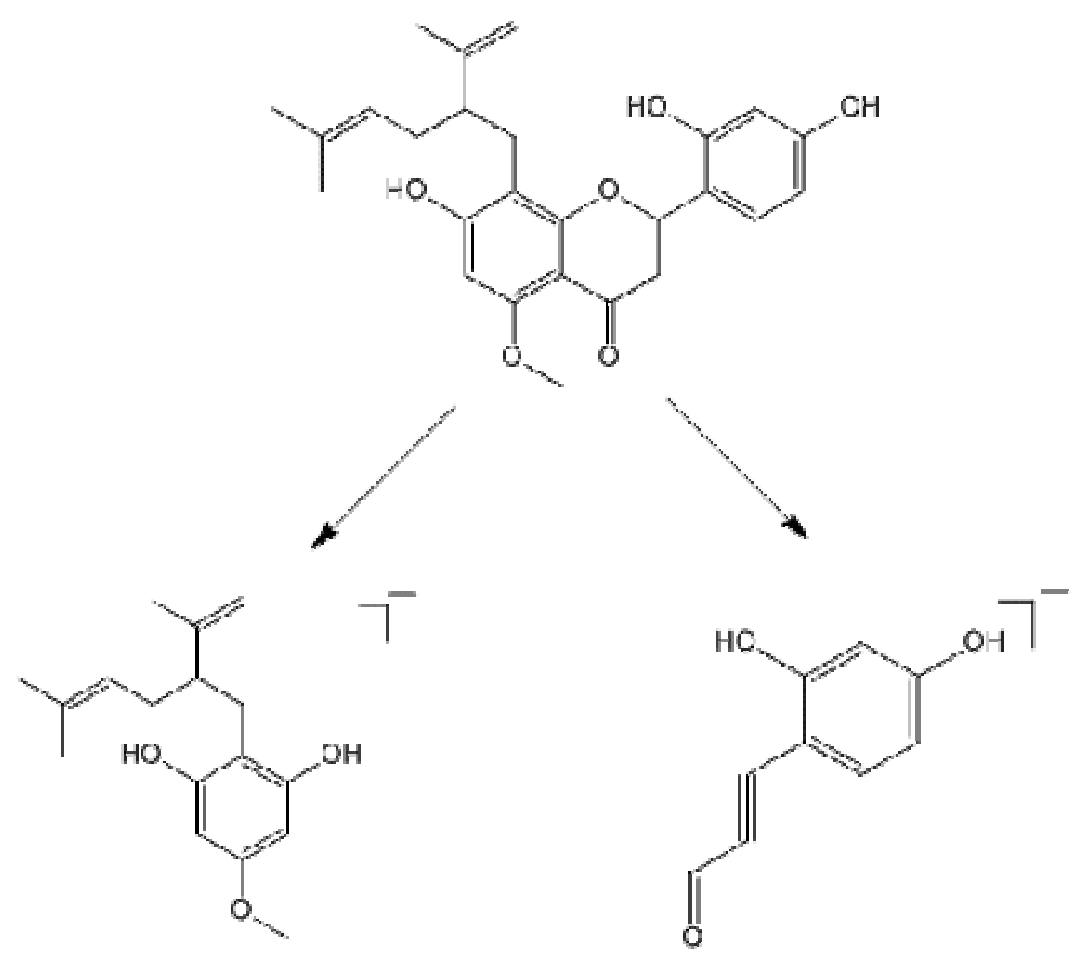

Figure 2: The proposed fragmentation pattern of kurarinone

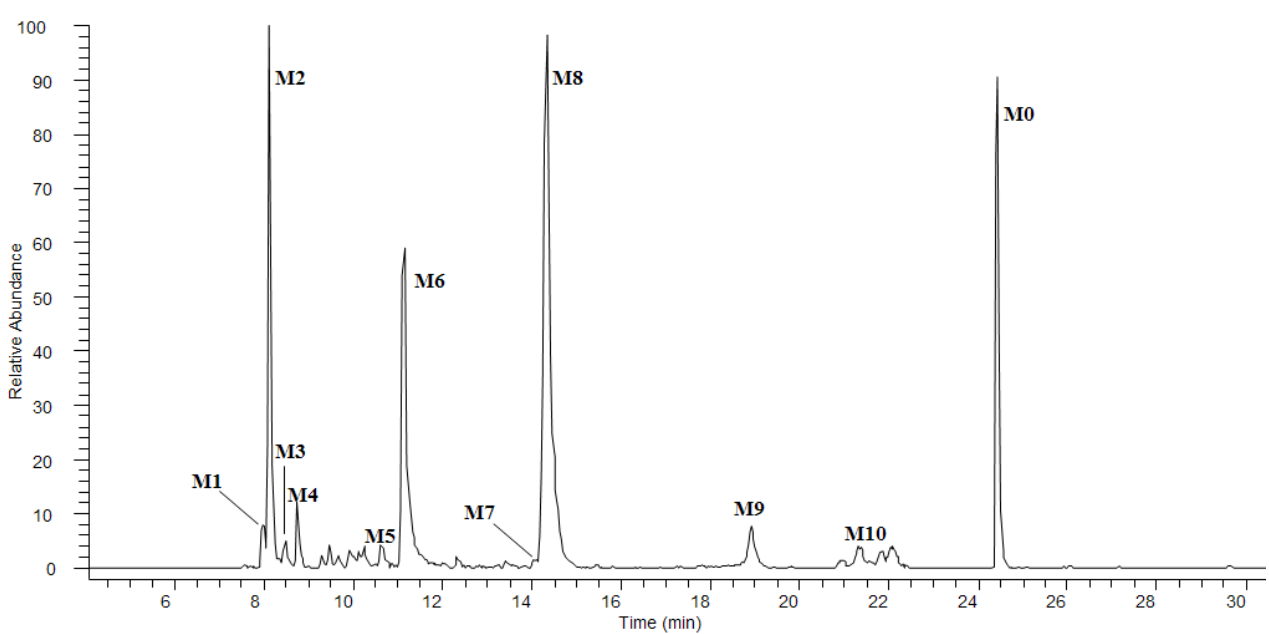

Figure 3: The total ion chromatograms of multiple metabolites in rats' urine

\section{Metabolite MO}

Metabolite M0 m/z 437.1958 (2.0 ppm, $\mathrm{C}_{26} \mathrm{H}_{29} \mathrm{O}_{6}$ ) eluted at $24.42 \mathrm{~min}$, and it was unambiguously identified as kurarinone by comparing the retention time, accurate mass measurement and fragment ions with the authentic reference material.

\section{Metabolites M7 - 10}

Metabolites M7, M8, M9, and M10 were eluted at $14.07,14.33,18.91$ and $21.32 \mathrm{~min}$, respectively. All of them yielded quasi-molecular ions of $\mathrm{m} / \mathrm{z}$
613.2269 (1.7 ppm, $\left.\mathrm{C}_{32} \mathrm{H}_{37} \mathrm{O}_{12}\right), 613.2265$ (2.4 ppm, $\left.\mathrm{C}_{32} \mathrm{H}_{37} \mathrm{O}_{12}\right)$, $613.2267\left(2.0 \mathrm{ppm}, \mathrm{C}_{32} \mathrm{H}_{37} \mathrm{O}_{12}\right)$, and $613.2268\left(1.9 \mathrm{ppm}, \mathrm{C}_{32} \mathrm{H}_{37} \mathrm{O}_{12}\right)$, respectively. In the $\mathrm{MS}^{2}$ spectra, there was an abundant product ion at $\mathrm{m} / \mathrm{z} 437$ that was produced by the neutral loss of $176 \mathrm{Da}$, indicating that they were glucuronide conjugates. Therefore, M7 - M10 were tentatively identified as glucuronidation of kurarinone.

\section{Metabolites M5 - 6}

Metabolites M5 and M6, with [M- H] ions at $\mathrm{m} / \mathrm{z}$ 789.2589 (1.4 ppm, $\left.\quad \mathrm{C}_{38} \mathrm{H}_{45} \mathrm{O}_{18}\right)$ and $\mathrm{m} / \mathrm{z}$

Trop J Pharm Res, June 2016; 15(6): 1302 
Table 1: Summary of kurarinone metabolites in rat urine

\begin{tabular}{|c|c|c|c|c|c|c|c|}
\hline Peak & $t_{R}$ & $\begin{array}{l}\text { Theoretical } \\
\text { mass } \underline{\mathrm{m} / \mathrm{z}}\end{array}$ & $\begin{array}{l}\text { Experimental mass } \\
\underline{\mathrm{m} / \mathrm{z}}\end{array}$ & $\begin{array}{l}\text { Error } \\
\text { (ppm) }\end{array}$ & $\begin{array}{l}\text { Formula [M- } \\
\mathrm{H}^{-}\end{array}$ & MS/MS fragment & Identification/reactions \\
\hline 1 & 7.97 & 629.2228 & 629.2218 & -1.7 & $\mathrm{C}_{32} \mathrm{H}_{37} \mathrm{O}_{13}$ & $\begin{array}{c}\mathrm{MS}^{2}[629]: 337(100), 453(71), 291(38) \\
233(12)\end{array}$ & $\begin{array}{l}\text { Glucuronidation and } \\
\text { Hydroxylation }\end{array}$ \\
\hline 2 & 8.11 & 629.2228 & 629.2219 & -1.5 & $\mathrm{C}_{32} \mathrm{H}_{37} \mathrm{O}_{13}$ & $\mathrm{MS}^{2}$ [629]: 291 (100), 453(98), 337(92) & $\begin{array}{l}\text { Glucuronidation and } \\
\text { Hydroxylation }\end{array}$ \\
\hline 3 & 8.48 & 629.2228 & 629.2221 & -1.2 & $\mathrm{C}_{32} \mathrm{H}_{37} \mathrm{O}_{13}$ & $\mathrm{MS}^{2}$ [629]: 453(100), 291(2) & $\begin{array}{l}\text { Glucuronidation and } \\
\text { Hydroxylation }\end{array}$ \\
\hline 4 & 8.74 & 629.2228 & 629.2217 & -1.9 & $\mathrm{C}_{32} \mathrm{H}_{37} \mathrm{O}_{13}$ & $\begin{array}{c}\mathrm{MS}^{2}[629]: 337(100), 453(64), 233(12) \\
291(5)\end{array}$ & $\begin{array}{l}\text { Glucuronidation and } \\
\text { Hydroxylation }\end{array}$ \\
\hline 5 & $\begin{array}{c}10.6 \\
2\end{array}$ & 789.2600 & 789.2589 & -1.4 & $\mathrm{C}_{38} \mathrm{H}_{45} \mathrm{O}_{18}$ & $\mathrm{MS}^{2}$ [789]: 613 (100), 293(68), 437(10) & Diglucuronidation \\
\hline 6 & $\begin{array}{c}11.1 \\
4\end{array}$ & 789.2600 & 789.2588 & -1.6 & $\mathrm{C}_{38} \mathrm{H}_{45} \mathrm{O}_{18}$ & $\mathrm{MS}^{2}$ [789]: $613(100), 437(8)$ & Diglucuronidation \\
\hline 7 & $\begin{array}{c}14.0 \\
7\end{array}$ & 613.2279 & 613.2269 & -1.7 & $\mathrm{C}_{32} \mathrm{H}_{37} \mathrm{O}_{12}$ & $\begin{array}{c}\mathrm{MS}^{2}[613]: 337(100), 437(92), 275(43) \\
419(21)\end{array}$ & Glucuronidation \\
\hline 8 & $\begin{array}{c}14.3 \\
3\end{array}$ & 613.2279 & 613.2265 & -2.4 & $\mathrm{C}_{32} \mathrm{H}_{37} \mathrm{O}_{12}$ & $\begin{array}{c}\mathrm{MS}^{2}[613]: 337(100), 437(85), 275(36) \\
419(14)\end{array}$ & Glucuronidation \\
\hline 9 & $\begin{array}{c}18.9 \\
1\end{array}$ & 613.2279 & 613.2267 & -2.0 & $\mathrm{C}_{32} \mathrm{H}_{37} \mathrm{O}_{12}$ & $\begin{array}{c}\mathrm{MS}^{2}[613]: 437(100), 293(78), 275(22), \\
419(8)\end{array}$ & Glucuronidation \\
\hline 10 & $\begin{array}{c}21.3 \\
2\end{array}$ & 613.2279 & 613.2268 & -1.9 & $\mathrm{C}_{32} \mathrm{H}_{37} \mathrm{O}_{12}$ & $\begin{array}{c}\mathrm{MS}^{2}[613]: 437(100), 293(68), 275(35) \\
419(4)\end{array}$ & Glucuronidation \\
\hline 0 & $\begin{array}{c}24.4 \\
2\end{array}$ & 437.1958 & 437.1950 & -2.0 & $\mathrm{C}_{26} \mathrm{H}_{29} \mathrm{O}_{6}$ & $\mathrm{MS}^{2}[437]: 161(100), 275(28)$ & Parent drug \\
\hline
\end{tabular}


789.2588 (1.6 ppm, $\mathrm{C}_{38} \mathrm{H}_{45} \mathrm{O}_{18}$ ) eluted at 10.62 and $11.14 \mathrm{~min}$, respectively.

In $\mathrm{MS}^{2}$ spectra, there was an abundant product ion at $\mathrm{m} / \mathrm{z} 613$ that was generated by the neutral loss of $176 \mathrm{Da}$, indicating that they were glucuronide conjugates. The $\mathrm{MS}^{3}$ of M5 and M6 possessed the same $\mathrm{MS}^{2}$ spectra as M7 - 10, suggesting that the core structure of M5 and M6 was the di - glucuronidation of kurarinone.

\section{Metabolites M1-4}

Metabolites M1, M2, M3, and M4 eluted at 7.97, $8.11,8.48$, and $8.74 \mathrm{~min}$, respectively. All of them yielded $[\mathrm{M}-\mathrm{H}]^{-}$ions at $\mathrm{m} / \mathrm{z} 629.2218(1.7$ ppm, $\mathrm{C}_{32} \mathrm{H}_{37} \mathrm{O}_{13}$ ), 629.2219 (1.5 ppm, $\mathrm{C}_{32} \mathrm{H}_{37} \mathrm{O}_{13}$ ), 629.2221 (1.2 ppm, $\mathrm{C}_{32} \mathrm{H}_{37} \mathrm{O}_{13}$ ), and 629.2217 (1.9 ppm, $\mathrm{C}_{32} \mathrm{H}_{37} \mathrm{O}_{13}$ ), respectively. In the $\mathrm{MS}^{2}$ spectra, there was an abundant product ion at $\mathrm{m} / \mathrm{z} 453$ that was produced by the neutral loss of $176 \mathrm{Da}$, indicating that they were glucuronide conjugates. The fragment ion at $\mathrm{m} / \mathrm{z} 453$ was present in the MS spectra, which was contained more $\mathrm{O}$ atoms more than $\mathrm{M} 0$ suggesting that it was a hydroxylation kurarinone ion. Therefore, M1 - M4 were tentatively identified as hydroxylation and glucuronidation of kurarinone.

\section{DISCUSSION}

Our results indicated that in vivo the kurarinone is typically transformed to non - toxic glucuronidation metabolites by phase II drugmetabolizing enzymes. In general, xenobiotics or drug compounds that are introduced into the body typically undergo metabolism, elimination and/or detoxification, then they are transformed to non-toxic metabolites by phase I and phase II drug-metabolizing enzymes. In general, conjugation with phase II DMEs (including sulfotransferases, UDP - glucuronosyltransferases and epoxide hydrolases) increases hydrophilicity, thereby enhancing excretion in the bile and/or the urine which consequently has a detoxification effect.

Although our results indicated that the UDP glucuronosyltransferases (UGT) play important roles in kurarinone metabolic pathways and a series of glucuronidation metabolic reactions after the oral administration kurarinone, metabolites M1 - 4 indicated that the hydroxylation of kurarinone also occurs, but it has a short half - life. The in vitro investigation of kurarinone metabolism in human liver microsomes also suggests that the hydroxylation of kurarinone was much slower than glucuronidation [11]. Our results suggested that kurarinone glucuronidation is considered to be more important than other metabolic pathway of kurarinone, and it is the major metabolic pathway in vivo.

\section{CONCLUSION}

The UPLC-LTQ-Orbitrap technique has been successfully applied to analyze the metabolic profile of orally administered kurarinone in Sparague-Dawley rat urine. A total of 10 metabolites and the parent drug were detected and identified based on accurate mass measurements, fragmentation patterns and chromatographic retention times.

To the best of our knowledge, this report is the first to describe the metabolism of kurarinone in vivo. The results indicate that kurarinone in vivo is typically transformed to non-toxic glucuronidation metabolites, and these findings help to characterize the metabolic profile of kurarinone in human liver microsomes in vitro by phase II drug-metabolizing enzymes.

\section{ACKNOWLEDGEMENT}

The authors greatly appreciate financial support from the National Foundation of Natural Sciences of China (Nos. 81303193 and 81403063).

\section{DECLARATIONS}

\section{Conflict of Interest}

No conflict of interest associated with this work.

\section{Contribution of Authors}

The authors declare that this work was done by the authors named in this article and all liabilities pertaining to claims relating to the content of this article will be borne by them.

\section{REFERENCES}

1. Wang Y, Curtis-Long MJ, Yuk HJ, Kim DW, Tan XF, Park $\mathrm{KH}$. Bacterial neuraminidase inhibitory effects of prenylated isoflavones from roots of Flemingia philippinensis. Bioorgan Med Chem 2013; 21: 63986404.

2. Ma HY, Zhou WS, Chu FJ, Wang D, Liang SW, Li S. HPLC fingerprint of flavonoids in Sophora flavescens and determination of five components. Chin $J$ Chin Mater Med 2013; 16: 2690-2695.

Trop J Pharm Res, June 2016; 15(6): 1304 
3. Kim BH, Na KM, Oh I, Song IH, Lee YS, Shin J, Kim TY. Kurarinone regulates immune responses through regulation of the JAK/STAT and TCR-mediated signaling pathways. Biochem Pharmacol 2013; 8: 11341144.

4. Chen L, Cheng $X$, Shi $W$, Lu Q, Go VL, Heber D, Ma $L$. Inhibition of growth of Streptococcus mutans, methicillinresistant staphylococcus aureus, and vancomycinresistant enterococci by kurarinone, a bioactive flavonoid isolated from Sophora flavescens. J Clin Microbiol 2005; 7: 3574-3575.

5. Lee SW, Lee HS, Nam JY, Kwon OE, Baek JA, Chang JS, Rho MC, Kim YK. Kurarinone isolated from Sophora flavescens Ait. inhibited MCP-1-induced chemotaxis. J Ethnopharmacol 2005; 3: 515-519.

6. Berghe WV, De Naeyer A, Dijsselbloem N, David JP, De Keukeleire D, Haegeman G. Attenuation of ERK/RSK2driven NFKB gene expression and cancer cell proliferation by kurarinone, a lavandulyl flavanone isolated from Sophora flavescens Ait.. Roots. Endocr Metab Immune Disord Drug Targets 2011; 11: 247-261.

7. De Naeyer A, Vanden Berghe W, Pocock V, Milligan S, Haegeman G, De Keukeleire D. Estrogenic and anticarcinogenic properties of kurarinone, a lavandulyl flavanone from the roots of Sophora flavescens. J Nat Prod 2004; 11: 1829-1832.

8. Seo OW, Kim JH, Lee KS, Lee KS, Kim JH, Won MH, Ha KS, Kwon YG, Kim YM. Kurarinone promotes TRAILinduced apoptosis by inhibiting NF-KB-dependent CFLIP expression in HeLa cells. Exp Mol Med 2012; 11: 653664.

9. Yu Q, Cheng N, Ni X. Identifying 2 prenylflavanones as potential hepatotoxic compounds in the ethanol extract of Sophora flavescens. J Food Sci 2013; 11: 1830-1834.

10. Sun M, Han J, Duan J, Cui Y, Wang T, Zhang W, Liu W, Hong J, Yao M, Xiong S, Yan X. Novel antitumor activities of Kushen flavonoids in vitro and in vivo. Phytother Res 2007; 21: 269-277.

11. Zhang $X$, Jiang $P$, Chen $P$, Cheng $N$. Metabolism of kurarinone by human liver microsomes and its effect on cytotoxicity. Pharm Biol 2015; 4: 619-627.

12. Xu C, Li CY, Kong AN. Induction of phase I, II and III drug metabolism/transport by xenobiotics. Arch Pharm Res 2006; 3: 249-268

13. Bayne K. Revised guide for the care and use of laboratory animals available. American Physiological Society. Physiologist 1996; 39 (4): 208-211. 